\title{
Inhibiting Interleukin 17 Can Ameliorate the Demyelination Caused by $A$. cantonensis via iNOS Inhibition
}

\author{
Feng Ying, ${ }^{1}$ Zheng Cunjing, ${ }^{2}$ Feng Feng, ${ }^{3}$ Wan Shuo, ${ }^{4}$ Zeng Xin, ${ }^{4}$ Xie Fukang, ${ }^{2}$ and \\ Wu Zhongdao ${ }^{4}$ \\ ${ }^{1}$ Medical School of South China University of Technology, Guangdong 510006, China \\ ${ }^{2}$ Histology and Embryology Department of Zhongshan School of Medicine, Sun Yat-sen University, Guangzhou 510080, China \\ ${ }^{3}$ The Department of Pharmacology and Toxicology, School of Pharmaceutical Sciences, Sun Yat-sen University, \\ Guangzhou 510080, China \\ ${ }^{4}$ Parasitology Department of Zhongshan School of Medicine, Sun Yat-sen University, Guangzhou 510080, China
}

Correspondence should be addressed to Xie Fukang; frankxie2000@yahoo.com and Wu Zhongdao; wuzhd@mail.sysu.edu.cn

Received 3 August 2017; Revised 18 September 2017; Accepted 2 October 2017; Published 18 December 2017

Academic Editor: Helieh S. Oz

Copyright @ 2017 Feng Ying et al. This is an open access article distributed under the Creative Commons Attribution License, which permits unrestricted use, distribution, and reproduction in any medium, provided the original work is properly cited.

Angiostrongylus cantonensis (A. cantonensis) is an important food-borne parasitic disease. Previous study showed that A. cantonensis infection can cause demyelination in the central nerve system, but the mechanism of action has not been understood. To explore the mechanism and to look for effective therapeutic methods, interleukin 17A (IL-17A) and iNOS expressions were detected during A. cantonensis infection. In addition, IL-17A-neutralizing antibody was applied to treat A. cantonensis-infected mice. In our results, we found that IL-17A and iNOS RNA expressions increased gradually in the process of $A$. cantonensis infection. When infected mice were treated with IL-17A-neutralizing antibody, the pathologic changes of demyelination alleviated obviously, followed with the elevation of myelin basic protein (MBP) in the brain. In addition, the iNOS expression of the brain in infected animals also showed a decrease in astrocytes. Our study provided evidence that IL-17A may take part in the demyelination caused by A. cantonensis and inhibiting IL-17A expression can ameliorate the pathologic changes of demyelination. Moreover, the decreasing of iNOS expression may be the key reason for the effect of IL-17A inhibition on demyelination caused by A. cantonensis.

\section{Introduction}

Angiostrongylus cantonensis (A. cantonensis) is an important cause of food-borne diseases and eosinophilic encephalitis in humans [1]. A. cantonensis is a parasitic nematode from rats which invades the central nerve system (CNS) and causes eosinophilic encephalitis or meningoencephalitis [2]. During this process, neurons in CNS appear with obvious demyelination [3-5] (MBP is one component of myelin sheath) [6]. However, the reason for demyelination associated with $A$. cantonensis infection has not been fully known.

Cytokines of the interleukin 17 (IL-17) family are uniquely placed on the border between immune cells and tissue. As seen in psoriatic skin lesions or in joints of rheumatoid arthritis patients, high levels of IL-17 have been detected in CNS during inflammatory responses. Previous study showed that IL-17-induced Act1-mediated signaling cascades in CNS resident cells (astrocytes, oligodendrocytes, and neurons) might coordinately mediate CNS inflammation, demyelination, and neurodegeneration $[7,8]$. But whether IL-17 is involved in the demyelination caused by Angiostrongylus cantonensis has never been studied.

Astrocytes probably represent the best-studied CNS resident cell type in the context of multiple sclerosis (MS) and EAE, which cause demyelination complications. Both human and mouse astrocytes (glial fibrillary acidic protein (GFAP) is the specific marker for astrocytes) express the IL-17RA, thereby allowing IL-17A ligation and consequently, the production of cytokines and chemokines, including IL-6, TNF $\alpha$, and CCL2 [9-11]. Inducible nitric oxide (iNOS) is 
involved in various physiological regulations and plays important roles in some CNS disease, such as brain ischemia, brain infections, and neurodegenerative diseases [12, 13].

In this study, we hypothesized that IL-17A expression is elevated during $A$. cantonensis infection and that antiIL-17A antibody can ameliorate the demyelination in infected animals. In this study, we report that IL-17A expressions were detected during A. cantonensis infection. Moreover, IL-17A-neutralizing antibody protects against demyelination caused by $A$. cantonensis infection. Our results showed that IL-17A and iNOS RNA expressions increased gradually in the process of A. cantonensis infection, and IL-17A inhibition alleviated the demyelination caused by $A$. cantonensis. Furthermore, we also report that IL-17A inhibition may decrease the production of iNOS, which might be the key reason for the curative effect of IL-17A-neutralizing antibody on the demyelination caused by A. cantonensis. These findings explore a role of IL-17A on the demyelination caused by $A$. cantonensis and provide a new potential alternative therapy for this disease.

\section{Materials and Methods}

2.1. Infection of Mice with A. cantonensis Larvae and IL-17 Antibody Injection. Mice infected with A. cantonensis larvae $\mathrm{BALB} / \mathrm{c}$ mice (20-40 g body weight) were purchased from the Animal Center Laboratory at Sun Yat-sen University (Guangzhou, China). The Institutional Animal Care and Use Committee approved all animal procedures. Larval collection: stage III larva (L3) of A. cantonensis were collected from giant African snails (Achatina fulica) via homogenization and digestion of minced snail tissue that was placed in a pepsin- $\mathrm{HCl}$ solution ( $\mathrm{pH} 2.0,500 \mathrm{IU}$ pepsin/gram tissue) and incubated at $37^{\circ} \mathrm{C}$ for $2 \mathrm{~h} . \mathrm{L} 3$ in the sediment were washed with phosphate-buffered saline (PBS) and counted under an anatomical microscope then given to experimental animals by gavage with $30 \mathrm{~L} 3$ per animal. The animals were divided into four groups: normal control group, normal control with IL-17 antibody, A. cantonensis infection group, and $A$. cantonensis infection group treated with IL-17 antibody. There are at least 5 mice in each group. The mice in the normal control treated with IL-17 antibody group and in the A. cantonensis infection treated with IL-17 antibody group were injected with IL-17-neutralizing antibody (per $0.05 \mathrm{mg} / \mathrm{kg} /$ day; eBioscience, USA) into the abdominal cavity at $0 \mathrm{~d}, 4 \mathrm{~d}, 8 \mathrm{~d}, 12 \mathrm{~d}, 16 \mathrm{~d}$, and $20 \mathrm{~d}$.

2.2. Transmission Electron Microscopy Observation. After anesthesia, the animals were euthanatized by transcardial perfusion with $4 \%$ paraformaldehyde. Mice' optic nerves were crosscut into $15 \mu \mathrm{m}$ sections at $-20^{\circ} \mathrm{C}$ and mounted on glass slides. Optic nerves were quickly dissected and postfixed overnight in $2.5 \%$ glutaraldehyde. Next, optic nerve fragments were postfixed in a solution containing $1 \%$ osmium tetroxide (Sigma-Aldrich), then fragments were dehydrated in acetone series and embedded in SPIN-PON resin. Resin polymerization was performed at $60^{\circ} \mathrm{C}$ for three days. Semithin sections ( $0.5 \mu \mathrm{m}$ thickness) were placed onto glass slides and stained with toluidine blue. Finally, demyelination detection was done by using a $300 \mathrm{kV}$ transmission electronic microscope (FEI, USA).

2.3. Immunofluorescence. After fixing with $4 \%$ paraformaldehyde, brain sections were cut into $15 \mu \mathrm{m}$ sections at $-20^{\circ} \mathrm{C}$ and mounted on glass slides. Then, sections were blocked with $3 \%$ bovine serum albumin (BSA) at room temperature for $1 \mathrm{~h}$ before incubation with rabbit anti-iNOS (Abcam, UK) and anti-GFAP (Sigma-Aldrich, USA) monoclonal antibody in $1 \%$ BSA at $4^{\circ} \mathrm{C}$ overnight. Sections were washed three times in PBS, incubated with fluorescein isothiocyanate- (TRITC-) labeled and FITC-labeled (for others) secondary antibody(Abcam, Cambridge, UK), diluted 1:500 in $1 \% \mathrm{BSA}$ at $37^{\circ} \mathrm{C}$ for $1 \mathrm{~h}$, and washed again in PBS. Then, DAPI (1:1000 dilution, Beyotime Biotechnology) stained the nucleus for $5 \mathrm{~min}$. Specimens stained without the primary antibody were used as negative controls. Then, the slides were observed under a confocal microscope.

2.4. RNA Isolation and Real-Time Quantitative PCR. Total RNA was extracted from the cerebrum with TRIzol reagent according to the manufacturer's instructions (Invitrogen). For cDNA synthesis, RNA was reverse transcribed with a PrimeScript RT reagent Kit (TaKaRa). The expression of the genes encoding IL-17A, and iNOS for mice by real-time PCR with SYBR Premix Ex Taq kit (TaKaRa). Relative quantification was applied to detect the mRNA expression of the above genes. The primer sequences as follows: $5^{\prime}$-TCATGTGGT GGTCCAGCTTTC-3', $3^{\prime}$-CTCAGACTACCTCAACCGTT CC- $5^{\prime}$ for IL-17A mice; $5^{\prime}$-CTGATGTTGCCATTGTTGG TG-3', $3^{\prime}$-CTTTGACGCTCGGAACTGTAG-5' for iNOS mice; and $5^{\prime}$-AAGAAGGTGGTGAAGCAGG-3'; $3^{\prime}$-GAAG GTGGAAGAGTGGGAGT-5 ${ }^{\prime}$ for GAPDH mice as an internal reference. Amplification of cDNA was performed on an ABI Prism 7900 HT cycler (Applied Biosystems).

2.5. Western Blot Analysis. The brain tissue of mice in different groups were washed twice with cold PBS and lysed in extraction buffer (20 mM HEPES, pH 7.4, $2 \mathrm{mM}$ EDTA, $50 \mathrm{mM}$ 5-glycerophosphate, $1 \mathrm{mM}$ dithiothreitol, $1 \mathrm{mM} \mathrm{Na} \mathrm{VO}_{4}$, $1 \%$ Triton X-100, and $10 \%$ glycerol) on ice. The lysates were centrifuged at $12,000 \mathrm{rpm}$ for $15 \mathrm{~min}$, and supernatants were collected. Protein (20-40 $\mu \mathrm{g})$ was separated by SDS-PAGE and then transferred onto a nitrocellulose membrane (Pall Corporation, Ann Arbor, MI, USA). Transferred blots were incubated sequentially with a blocking agent (5\% nonfat milk in TBS), and an anti-MBP antibody (1:125 dilution, Abcam, $\mathrm{UK}$ ) and a HRP-conjugated secondary antibody (for $1 \mathrm{~h}$ at room temperature) were developed by the enhanced chemifluorescence detection kit on Hyperfilm (Fuji, Japan) according to the manufacturer's directions. The same blots were subsequently stripped and reblotted with internal referring antibodies $\beta$-actin and $\beta$-tubulin (Sigma-Aldrich, USA). Graphs of blots were obtained in the linear range of detection and were quantified for the level of specific induction by Image J System.

2.6. Statistical Analysis. One-way ANOVA was used to compare data of real-time PCR and graphs of blots in western 

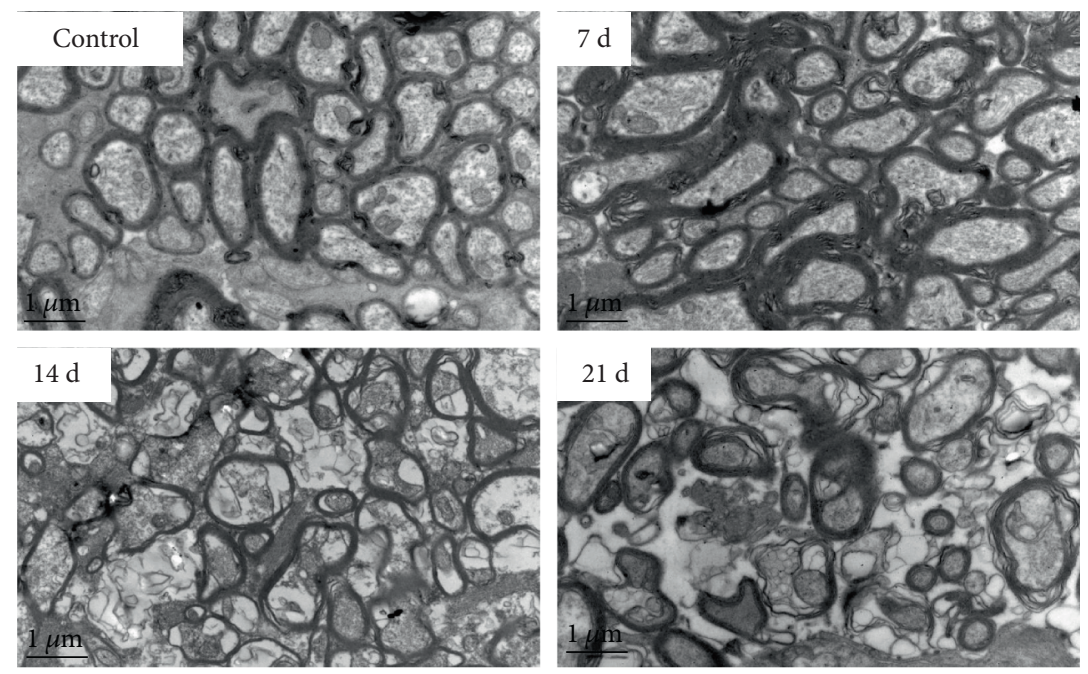

(a)

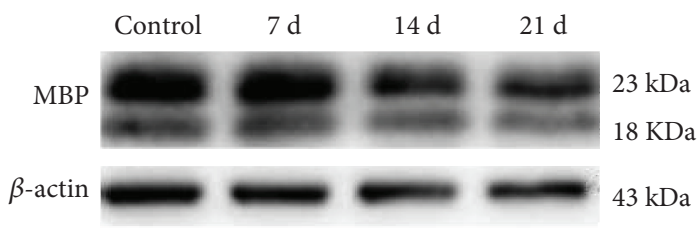

(b)

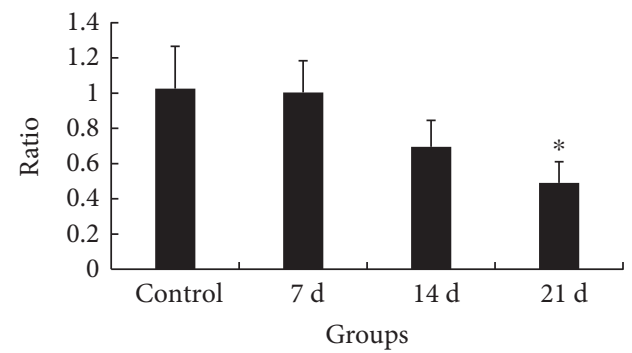

(c)

FIgURE 1: A. cantonensis infection caused demyelination in infected mice. (a) Transmission electronic image of the optic nerve at $0 \mathrm{~d}, 7 \mathrm{~d}, 14 \mathrm{~d}$, and $21 \mathrm{~d}$ after $A$. cantonensis infection. Prominent demyelination (the black arrows point) can be observed in $14 \mathrm{~d}$ and $21 \mathrm{~d}$. Scale bar $=1 \mu \mathrm{m}$. (b) The MBP protein expression of the brain via western blotting at $0,7,14$, and 21 days after A. cantonensis infection. (c) The relative ration of MBP protein expression ( $\beta$-actin was as the internal reference). These expressions decreased as time extended and had obvious difference with the control in $21 \mathrm{~d}$ of infection. Numerical results are presented as mean \pm SEM. $n=3$ animals per group, at least three fields were analyzed per section in at least two sections not next to each other per animal. * represents statistically significant values when compared with the normal control $(P<0.05)$.

blotting among different groups. Statistics was performed using IBM SPSS statistics 19 (SPSS Inc., USA). $P<0.05$ was considered statistically significant.

\section{Results}

3.1. Demyelination Is of Serious Pathological Change in the Brain Tissue of Mice with A. cantonensis Infection. A. cantonensis invaded the central nerve system and caused demyelination. The resulting images of the transmission electronic microscope of the optic nerves showed that demyelination was obvious at $14 \mathrm{~d}$ and became serious on $21 \mathrm{~d}$ of infection of A. cantonensis (Figure 1(a)). Moreover, the MBP (myelin basic protein) expression also decreased gradually during A. cantonensis infection (Figures 1(b) and 1(c)). The above results proved that $A$. cantonensis can cause demyelination in the brain.

3.2. Increase of IL-17A and iNOS Is Significant in the Brain of the Infected Mice. We further explored whether the expression of IL-17A is altered in the process during A. cantonensis infection. We found that IL-17A RNA expression increased with the extension of the infection (Figure 2(a)). At the same time, the RNA expression of iNOS also increased gradually and peaked at $21 \mathrm{~d}$ of infection (Figure 2(b)). These findings suggest that IL-17A and iNOS may correlate with demyelination caused by $A$. cantonensis.

3.3. IL-17A Inhibition Can Lighten the Demyelination in the Brain of the Infected Mice. TEM and MBP protein expressions were applied to detect the alteration of demyelination. When A. cantonensis infected the mice for $21 \mathrm{~d}$, TEM showed obvious demyelination. Moreover, MBP expression also decreased distinctly. After IL-17A antibody was injected in experimental mice, the results showed that IL-17A inhibition can restore the demyelination caused by A. cantonensis to normal levels (Figure 3(a)). In addition, levels of MBP and expression were elevated after IL-17A inhibition of A. cantonensis in infected mice but had no effect on normal mice (Figures $3(\mathrm{~b})$ and $3(\mathrm{c}))$. These results displayed that IL-17A may be the key element for demyelination caused by $A$. cantonensis.

3.4. IL-17A Inhibition Can Cause the Downregulation of iNOS in Astrocytes in the Brain of the Infected Mice. From 
IL-17A

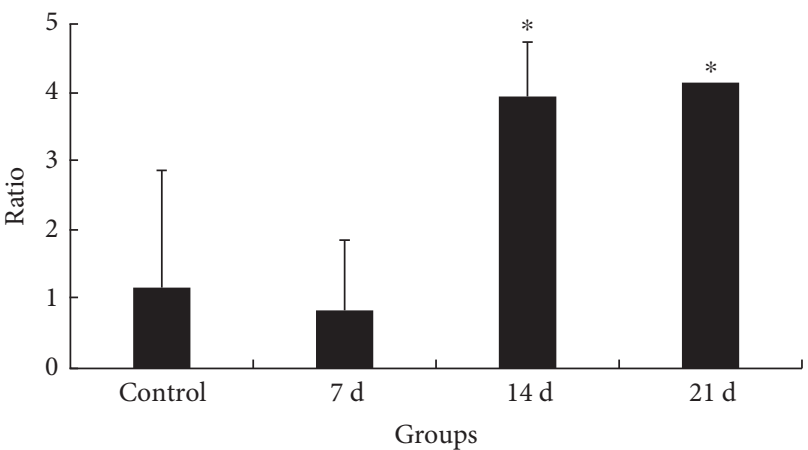

(a)

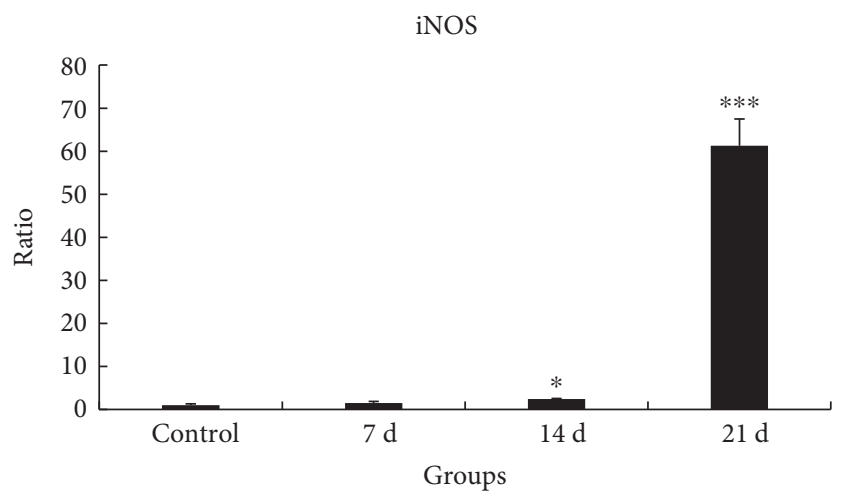

(b)

Figure 2: A. cantonensis induced the elevation of IL-17A and iNOS. The RNA expression of IL-17A and iNOS at $0 \mathrm{~d}, 7 \mathrm{~d}, 14 \mathrm{~d}$, and $21 \mathrm{~d}$ after A. cantonensis infection (GAPDH was the internal reference for real-time PCR). (a) Expression of IL-17A increased gradually. $n=3$ animals per group. (b) RNA expression of iNOS, which also elevated with infection time obviously. $*$ and $* * *$ represent statistically significant values when compared with normal control $(P<0.05$ and $P<0.001$, resp.).
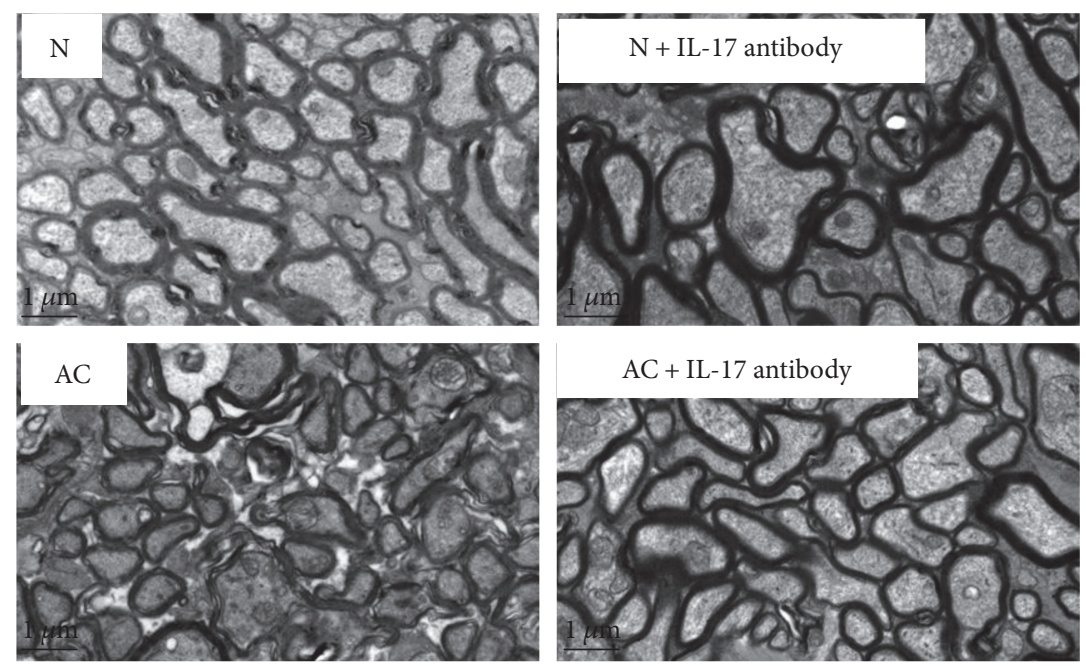

(a)

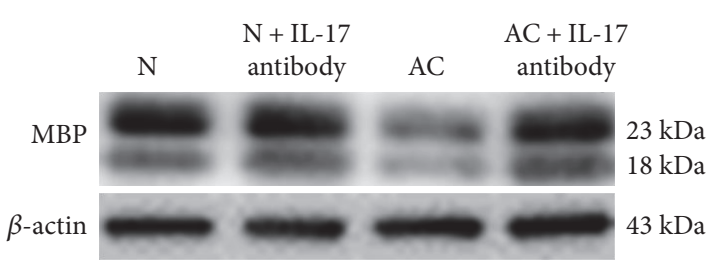

(b)

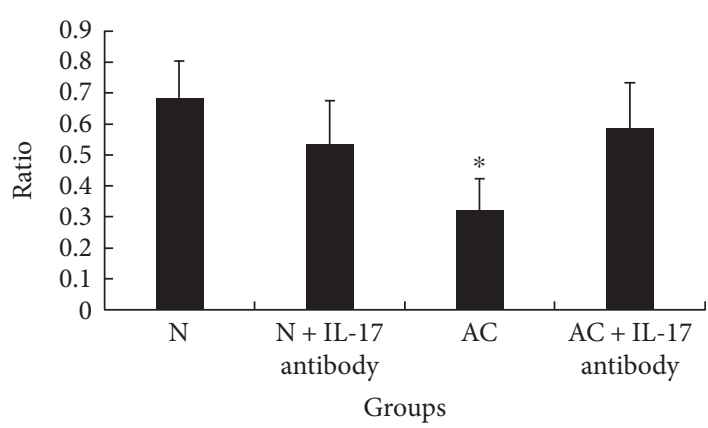

(c)

FIGURE 3: IL-17A inhibition can ameliorate the demyelination caused by A. cantonensis. (a) TEM picture of the optic nerve in the normal group, $21 \mathrm{~d}$ A. cantonensis infection group, normal mice with IL-17A antibody group, and $21 \mathrm{~d}$ A. cantonensis infection with IL-17A antibody group. IL-17A inhibition can restore the demyelination caused by A. cantonensis to the normal level. Scale bar $=1 \mu \mathrm{m}$. (b) The MBP protein expression of the brain via western blotting in the normal group, $21 \mathrm{~d}$ A. cantonensis infection group, normal mice with IL-17A antibody group, and $21 \mathrm{~d}$ A. cantonensis infection with IL-17A antibody group. (c) The semiquantification of MBP protein expression via western blotting in different groups ( $\beta$-actin as the internal reference). IL-17A inhibition had no effect on MBP expression of the brain in the normal group but increased MBP expression in the $21 \mathrm{~d} A$. cantonensis infection group. $n=3$ animals per group, at least three fields were analyzed per section in at least two nonadjacent sections per animal. * represents statistically significant values when compared with normal control $(P<0.05)$. 

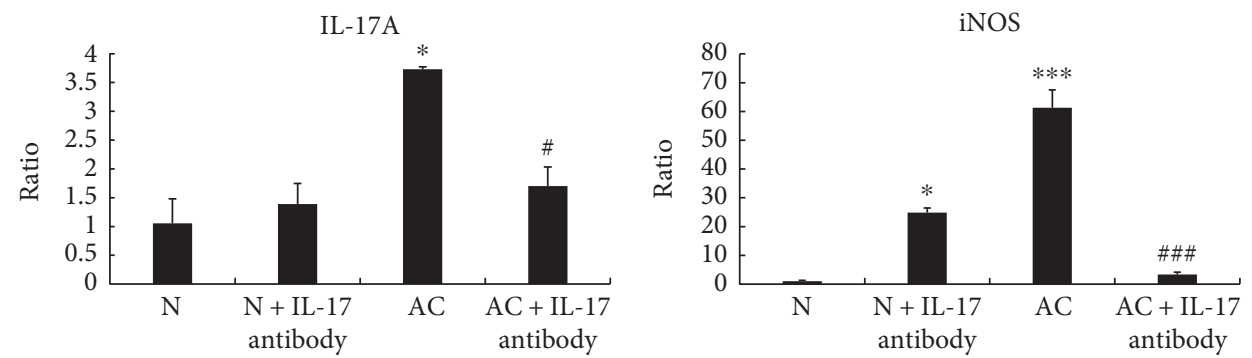

(a)
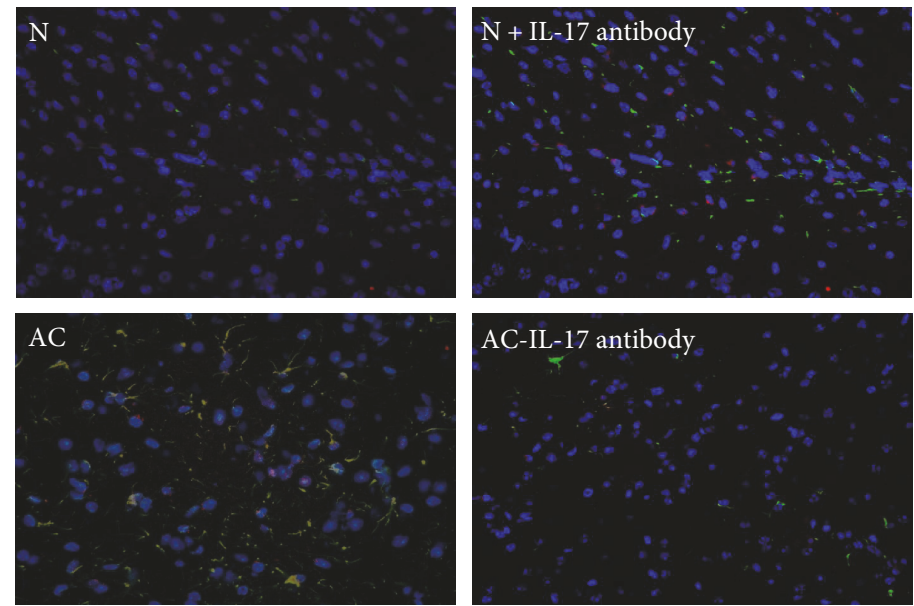

(b)

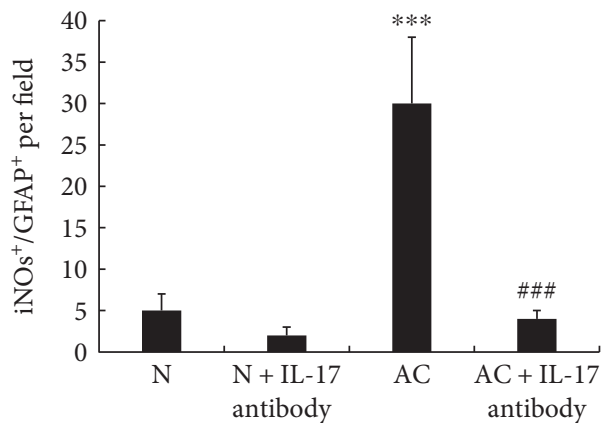

(c)

FIgURE 4: IL-17A inhibition causes the downregulation of iNOS in astrocytes after A. cantonensis infection. (a) The RNA expression of IL-17A and iNOS in the normal group, the $21 \mathrm{~d}$ A. cantonensis infection group, the normal mice with IL-17A antibody group, and the 21 $\mathrm{d}$ A. cantonensis infection with IL-17A antibody group. After IL-17A antibody was injected, the RNA expression of IL-17A was decreased in the A. cantonensis-infected group on day 21 but it had no effect on the normal group. IL-17A antibody increased the RNA expression of iNOS in the normal group, but levels were decreased in the $21 \mathrm{~d}$ A. cantonensis infection group. GAPDH was the internal reference for real-time PCR. (b) Brain sections stained with iNOS (red) and GFAP (green). Yellow color represented the double staining of iNOS and GFAP. (c) Percentage of $\mathrm{iNOS}^{+} / \mathrm{GFAP}^{+}$cells per field of the brain section. ${ }^{*} P<0.05,{ }^{* * *} P<0.001$, compared with the normal group; ${ }^{*} P<0.05$,

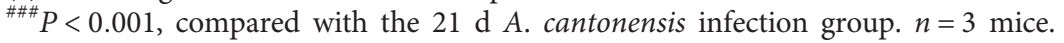

above results, we found that IL-17A inhibition can alleviate demyelination caused by $A$. cantonensis, but the mechanism was still unknown. To resolve this question, real-time PCR and immunofluorescence were applied to examine the expression of IL-17A and iNOS. Our results showed when A. cantonensis infected the mice for $21 \mathrm{~d}, \mathrm{IL}-17 \mathrm{~A}$ and iNOS elevated obviously. IL-17A-neutralizing antibody can decrease the expression of IL-17A, which proved that the inhibition of IL-17A in A. cantonensis-infected mice was effective, but it cannot influence the IL-17A expression of normal animals. More importantly, iNOS expression was also decreased obviously during IL-17A inhibition. Moreover, we found that when IL-17A-neutralizing antibody was applied, iNOS expression in normal mice was elevated (Figure 4(a)). The results of immunofluorescence also proved that when $A$. cantonensis infected the mice for $21 \mathrm{~d}$, the double labeling of iNOS and GFAP (astrocytes marker) both increased, whereas IL-17A inhibition can decrease the protein expression of iNOS in astrocytes (Figures 4(b) and 4(c)). The above results showed that IL-17A inhibition may alleviate demyelination caused by $A$. cantonensis through decreasing the iNOS expression in astrocytes.

\section{Discussion}

A. cantonensis is an important cause of food-borne diseases in humans. A. cantonensis infection is endemic in the Pacific Islands, Southeast Asia, China, and Hawaii. Sporadic infections are reported in Southern United States and Florida [14]. Outbreaks of eosinophilic meningitis have been reported due to the consumption of raw snails harboring L3 or associated with the consumption of contaminated raw vegetable juice in Taiwan $[15,16]$. Rat is a known definitive host which can tolerate the worms. In large numbers (240), A. cantonensis cause significant cardiovascular and neurological impairment in rats [17].

In this study, we detected IL-17A expression in the brain tissue of the infected mice and applied IL-17A-neutralizing antibody to treat the infected mice to investigate the process of brain demyelination. Our findings demonstrated that A. cantonensis infection promotes the IL-17A expression in the brain as well as IL-17A inhibition to alleviate the demyelination caused by A. cantonensis in mice. Moreover, IL-17A inhibition may decrease the production of iNOS, which might be the reason for the curative effect for 
IL-17A-neutralizing antibody on demyelination caused by A. cantonensis. The evidence was as follows.

Firstly, TEM detection of the optic nerve and western blotting of MBP proved that $A$. cantonensis infection actually caused demyelination. Moreover, the RNA expression of IL-17A and iNOS increased gradually following with the prolonged $A$. cantonensis infection. IL-17 is a mediator of communication between immune cells and tissues. IL-17 is the founding member of a family of 6 cytokines, IL-17A-F, and IL-17A is the most investigated one. IL-17A has recently emerged as an attractive target, especially for the treatment of $\mathrm{T}$ cell-mediated autoimmune diseases $[18,19]$. Previous study showed that IL-17A expression alone was able to activate glial cells and enhance neuroinflammatory responses, thus showing that CNS cells express a functional IL-17RA/C receptor complex [6]. In our study, IL-17A expression elevated with demyelination during A. cantonensis infection. Similarly, we observed elevation of iNOS associated with demyelination in experimental animals. These findings indicate that IL-17A along with iNOS may take part in the demyelination caused by A. cantonensis.

Next, the IL-17A-neutralizing antibody was administered in the infected mice to explore the function of IL-17A on demyelination caused by A. cantonensis. Our results proved that IL-17A inhibition can alleviate demyelination obviously observed via TEM of the optic nerve and western blotting of MBP after A. cantonensis infection. In the previous study, there were strong indications pointing to a role for IL-17 in the pathogenesis of MS and EAE, major causes of demyelination diseases $[20,21]$. As a result, we applied IL-17Aneutralizing antibody to treat the demyelination caused by A. cantonensis and the effect proved our presumption.

Finally, we explored the mechanism by which IL-17Aneutralizing antibody alleviated demyelination caused by A. cantonensis. Our results showed that IL-17A-neutralizing antibody inhibited the RNA expression of IL-17A and also decreased the production of iNOS induced by A. cantonensis infection. Moreover, IL-17A-neutralizing antibody also inhibited the protein expression of iNOS in astrocytes. Astrocytes probably represent the best-studied CNS resident cell type in the context of MS and EAE. Both human and mouse astrocytes express IL-17RA, thereby allowing for IL-17A ligation and consequently, the production of cytokines and chemokines, including IL-6, TNF $\alpha$, and CCL2 [6, 7]. iNOS is involved in various physiological regulations and plays important roles in some CNS disease, such as brain ischemia, brain infections, and neurodegenerative diseases $[22,23]$. As a result, we speculated that IL-17A-neutralizing antibody alleviated demyelination via inhibiting iNOS production in astrocytes. Previous study showed that exogenous IL-17A significantly induced iNOS expression and hence cardiomyocyte apoptosis [24]. Our study was partly in accordance with these findings. To the best of our knowledge, there has been no previous report that IL-17A inhibition would decrease the production of iNOS in demyelination caused by $A$. cantonensis. In this line, we will further investigate iNOS using knockout mice in future studies.

In conclusion, IL-17A may play important roles in demyelination caused by $A$. cantonensis and the present study suggested that IL-17A-neutralizing antibody may be an effective method to treat the demyelination caused by $A$. cantonensis. Furthermore, iNOS inhibition is the possible mechanism for the therapeutic effect. As a result, our study provides a new potential alternative therapy for demyelination caused by $A$. cantonensis.

\section{Conflicts of Interest}

The authors declare that they have no competing interests.

\section{Authors' Contributions}

Feng Ying, Zheng Cunjing, Feng Feng, and Wan Shuo carried out the experiments and performed the statistical analyses. Feng Ying drafted the manuscript. Wu Zhongdao and Xie Fukang conceived the study and coordinated the project. All authors read and approved the final manuscript. Feng Ying and Zheng Cunjing contributed equally to this work.

\section{Acknowledgments}

This work was supported by the National Natural Science Foundation of China (81401688, 81271855, and 81261160324), the National Research and Development Plan of China (no. 2016YFC1200500), the Central College basic research operating funding of the South China University of Technology (2017MS090), the South China University of Technology scientific research funding (D6172910), and the South China University of Technology School of Medicine scientific research project funding (yxy2016007).

\section{References}

[1] J. J. Wang, L. Y. Chung, R. J. Lin, J. D. Lee, C. W. Lin, and C. M. Yen, "Eosinophilic meningitis risk associated with raw Ampullarium canaliculatus snails consumption," The Kaohsiung Journal of Medical Sciences, vol. 27, no. 5, pp. 184-189, 2011.

[2] Q. P. Wang, Z. D. Wu, J. Wei, R. L. Owen, and Z. R. Lun, "Human Angiostrongylus cantonensis: an update," European Journal of Clinical Microbiology \& Infectious Diseases, vol. 31, pp. 389-395, 2012.

[3] K. Y. Lin, K. M. Chen, K. P. Lan, H. H. Lee, and S. C. Lai, "Alterations of myelin proteins in inflammatory demyelination of BALB/c mice caused by Angiostrongylus cantonensis," Veterinary Parasitology, vol. 171, no. 1-2, pp. 74-80, 2010.

[4] Y. Feng, X. Zeng, W. H. Li et al., "The pathogenesis of optic neuritis caused by Angiostrongylus cantonensis in BALB/C mice," Parasites \& Vectors, vol. 7, pp. 339-351, 2014.

[5] F. Feng, Y. Feng, Z. Liu et al., "Effects of albendazole combined with TSII-A (a Chinese herb compound) on optic neuritis caused by Angiostrongylus cantonensis in BALB/c mice," Parasites \& Vectors, vol. 25, no. 8, pp. 606-616, 2015.

[6] E. Meinl and R. Hohlfeld, "Immunopathogenesis of multiple sclerosis: MBP and beyond," Clinical \& Experimental Immunology, vol. 128, no. 3, pp. 395-397, 2002.

[7] A. Waisman, J. Hauptmann, and T. Regen, "The role of IL-17 in CNS diseases," Acta Neuropathologica, vol. 129, pp. 625637, 2015. 
[8] B. S. Kim, Y. J. Park, and Y. Chung, "Targeting IL-17 in autoimmunity and inflammation," Archives of Pharmacal Research, vol. 39, no. 11, pp. 1537-1547, 2016.

[9] D. W. Luchtman, E. Ellwardt, C. Larochelle, and F. Zipp, "IL-17 and related cytokines involved in the pathology and immunotherapy of multiple sclerosis: current and future developments," Cytokine \& Growth Factor Reviews, vol. 25, no. 4, pp. 403-413, 2014.

[10] Y. Zhang, R. Huang, Y. Zhang et al., "IL-17 induces MIP- $1 \alpha$ expression in primary mouse astrocytes via TRPC channel," Inflammopharmacology, vol. 24, no. 1, pp. 33-42, 2016.

[11] G. Elain, K. Jeanneau, A. Rutkowska, A. K. Mir, and K. K. Dev, "The selective anti-IL17A monoclonal antibody secukinumab (AIN457) attenuates IL17A-induced levels of IL6 in human astrocytes," Glia, vol. 62, no. 5, pp. 725-735, 2014.

[12] B. Moran, C. M. Sweeney, R. Hughes et al., "Hidradenitis suppurativa is characterised by dysregulation of the Th17:Treg cell axis, which is corrected by anti-TNF therapy," The Journal of Investigative Dermatology, vol. 137, no. 11, pp. 2389-2395, 2017.

[13] R. Pannu and I. Singh, "Pharmacological strategies for the regulation of inducible nitric oxide synthase: neurodegenerative versus neuroprotective mechanisms," Neurochemistry International, vol. 49, no. 2, pp. 170-182, 2006.

[14] H. D. Stockdale Walden, J. D. Slapcinsky, S. Roff et al., "Geographic distribution of Angiostrongylus cantonensis in wild rats (Rattus rattus) and terrestrial snails in Florida, USA," PLoS One, vol. 12, no. 5, article e0177910, 2017.

[15] L. Ji, X. Yiyue, H. Xujin et al., "Study on the tolerance and adaptation of rats to Angiostrongylus cantonensis infection," Parasitology Research, vol. 116, no. 7, pp. 1937-1945, 2017.

[16] H. C. Tsai, S. S. Lee, C. K. Huang, C. M. Yen, E. R. Chen, and Y. C. Liu, "Outbreak of eosinophilic meningitis associated with drinking raw vegetable juice in southern Taiwan," The American Journal of Tropical Medicine and Hygiene, vol. 71, no. 2, pp. 222-226, 2004.

[17] P. Fasching, M. Stradner, W. Graninger, C. Dejaco, and J. Fessler, "Therapeutic potential of targeting the Th17/Treg axis in autoimmune disorders," Molecules, vol. 22, no. 1, 2017.

[18] E. Volpe, L. Battistini, and G. Borsellino, "Advances in T helper 17 cell biology: pathogenic role and potential therapy in multiple sclerosis," Mediators of Inflammation, vol. 2015, Article ID 475158, 11 pages, 2015.

[19] L. Brockmann, A. D. Giannou, N. Gagliani, and S. Huber, "Regulation of TH17 cells and associated cytokines in wound healing, tissue regeneration, and carcinogenesis," International Journal of Molecular Sciences, vol. 18, no. 5, 2017.

[20] A. Ghaffarinia, S. Parvaneh, C. Jalili, F. Riazi-Rad, S. Yaslianifard, and N. Pakravan, "Immunomodulatory effect of chymotrypsin in CNS is sex-independent: evidence of anti-inflammatory role for IL-17 in EAE," Iranian Journal of Allergy, Asthma, and Immunology, vol. 15, no. 2, pp. 145$155,2016$.

[21] D. W. Wojkowska, P. Szpakowski, and A. Glabinski, "Interleukin 17A promotes lymphocytes adhesion and induces CCL2 and CXCL1 release from brain endothelial cells," International Journal of Molecular Sciences, vol. 18, p. 5, 2017.

[22] C. Rapôso, R. L. Luna, A. K. Nunes, R. Thomé, and C. A. Peixoto, "Role of iNOS-NO-cGMP signaling in modulation of inflammatory and myelination processes," Brain Research Bulletin, vol. 104, pp. 60-73, 2014.
[23] I. Stevanovic, M. Ninkovic, I. Stojanovic, S. Ljubisavljevic, S. Stojnev, and D. Bokonjic, "Role of iNOS-NO-cGMP signaling in modulation of inflammatory and myelination processes," Brain Research Bulletin, vol. 104, pp. 60-73, 2014.

[24] S. A. Su, D. Yang, W. Zhu et al., "Interleukin-17A mediates cardiomyocyte apoptosis through Stat3-iNOS pathway," Biochimica et Biophysica Acta (BBA) - Molecular Cell Research, vol. 1863, no. 11, pp. 2784-2794, 2016. 


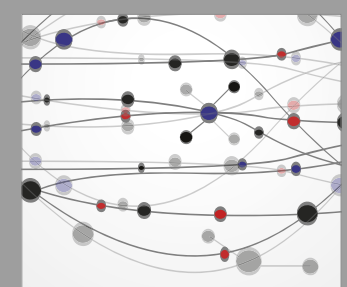

The Scientific World Journal


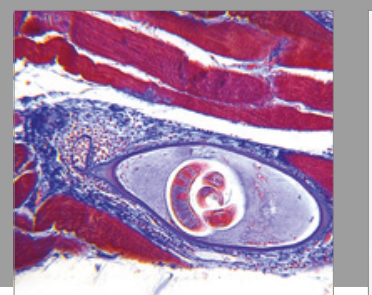

Gastroenterology Research and Practice
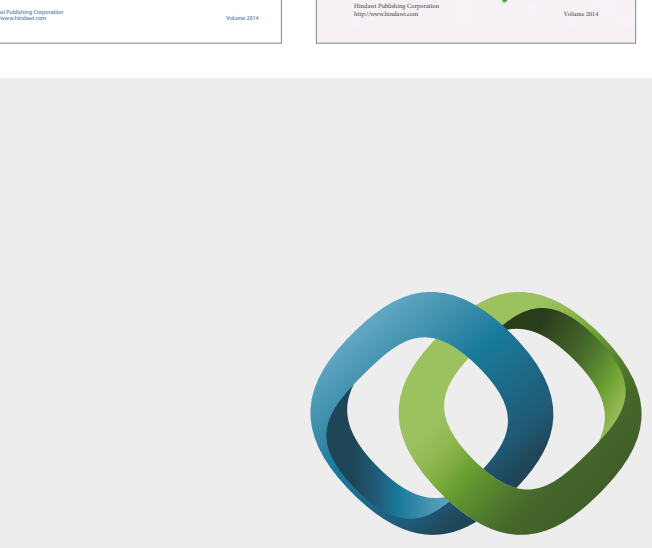

\section{Hindawi}

Submit your manuscripts at

https://www.hindawi.com
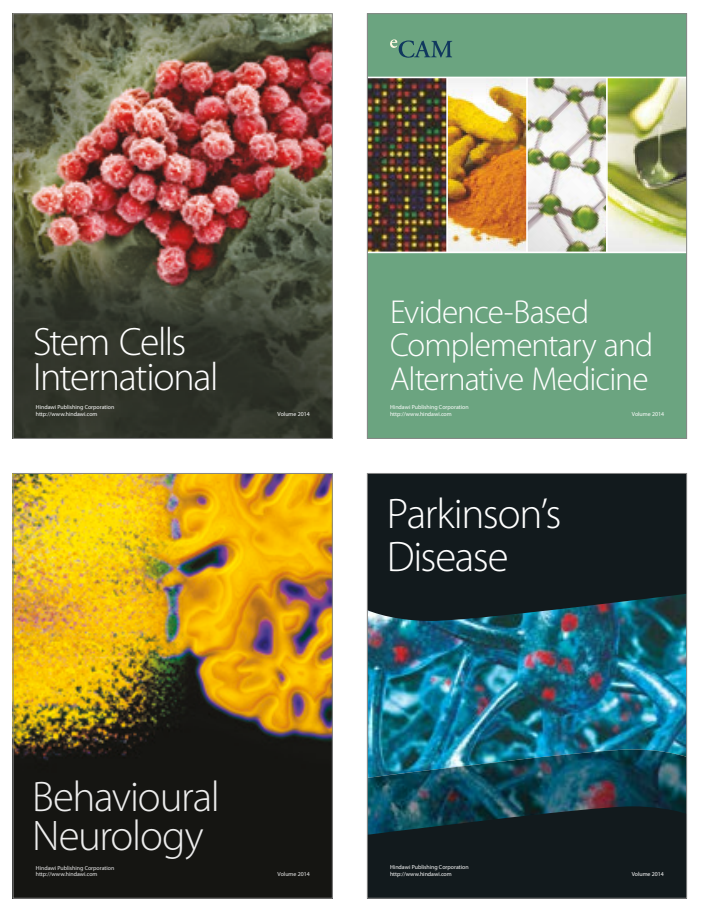
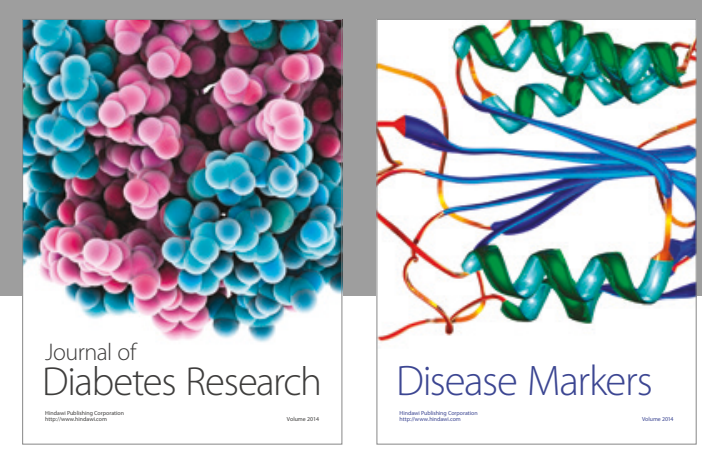

Disease Markers
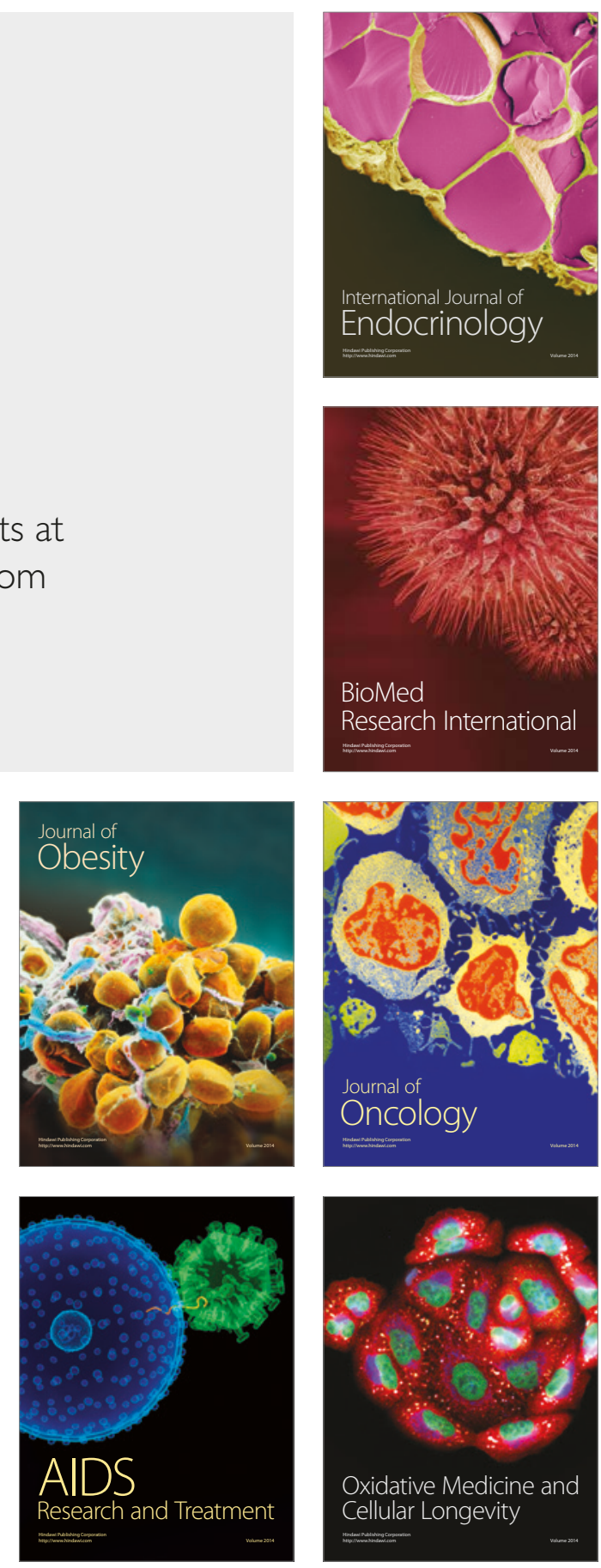